BULLETIN OF THE

AMERICAN MATHEMATICAL SOCIETY

Volume 77, Number 4, July 1971

\title{
A NONSMOOTHABLE KNOT
}

\author{
BY RICHARD K. LASHOF
}

Communicated by Saunders Mac Lane, February 1, 1971

In this note we prove the existence of a locally flat topological embedding of $S^{3}$ in $S^{5}$, which is not equivalent to a smooth embedding under homeomorphism of $S^{5}$.

The proof depends heavily on the results of Cappell and Shaneson [CS]. In their paper they describe a homotopy equivalence $h$ of $M=S^{3} \times S^{1} \# r\left(S^{2} \times S^{2}\right)$ onto itself, which is not homotopic to a diffeomorphism (Proposition 3.2 and Example 1.6). It is not known whether $h$ can be taken to be a homeomorphism; however $h \times 1: M$ $\times S^{1} \rightarrow M \times S^{1}$ is homotopic to a homeomorphism but not a diffeomorphism. Thus the homeomorphism, say $k: M \times S^{1} \rightarrow M \times S^{1}$, represents the nontrivial element $\alpha \in \pi_{3}$ (Top/PL) $=Z_{2}$, i.e., the obstruction in $H^{3}\left(M \times S^{1} ; \pi_{3}\right.$ (Top/PL)) to smoothing $k$, (see [KS] and Remark 2 ).

Using the trivial normal bundle of $S^{3} \subset S^{3} \times S^{1} \# r\left(S^{2} \times S^{2}\right)$, we have a smooth embedding of $S^{3} \times R$ in $M$ and $S^{3} \times R^{2}$ in $M \times R$. Now $k$ has a covering map $\hat{k}: M \times R \rightarrow M \times R$, also representing $\alpha$, since the obstruction depends only on $k$ in a neighborhood of $S^{3}$. We will define a smooth embedding $i: M \times R \rightarrow S^{5}$; then $f=i \circ \hat{k} \mid S^{3} \times R^{2}: S^{3} \times R^{2} \rightarrow S^{5}$ will also represent $\alpha$, and we will show that $f \mid S^{3} \times 0$ is the desired locally flat embedding.

To define $i$, take a standard embedding of $S^{3}$ in $S^{5}$, and then its normal sphere bundle $S^{3} \times S^{1}$ will be smoothly embedded in $S^{5}$ with a trivial normal bundle. We may add trivial 2-handles to $S^{3} \times S^{1}$ $\times I \subset S^{5}$, to embed $M$ (in fact, the cobordism from $S^{3} \times S^{1}$ to $M$ ) in $S^{5}$ with a trivial normal bundle. Thus we get our smooth embedding $i: M \times R \rightarrow S^{5}$.

Now suppose there exists a homeomorphism $g: S^{5} \rightarrow S^{5}$ such that $g \circ f \mid S^{3} \times 0$ is smooth. By the uniqueness of codimension two normal bundles [K], we may assume $g \circ f: S^{3} \times R^{2} \rightarrow S^{5}$ is smooth. ${ }^{1}$ On the other hand, any homeomorphism of $S^{5}$ is isotopic to a diffeomorphism [KS]; say $g$ is isotopic to the diffeomorphism $d$. Then $d^{-1} \circ g \circ f$ is

AMS 1969 subject classifications. Primary 5701, 5705, 5520, 5478.

Key words and phrases. Knot, locally flat embedding, nonsmoothable, homeomorphism of a manifold.

${ }^{1}$ Reference to [K] may be avoided as follows: $g \circ f\left(S^{3} \times R^{2}\right)$ is diffeomorphic to $S^{3} \times R^{2}$ by engulfing, and hence is the trivial smoothing. Therefore, $g \circ f$ is ambient isotopic to a smooth embedding; and thus we may assume $g$ of is smooth. 
smooth, but $d^{-1} \circ \mathrm{g}$ is isotopic to the identity. Therefore $f$ is isotopic to a smooth embedding, and represents the trivial class in $\pi_{3}$ (Top/PL). Contradiction. Thus we have proved:

THEOREM. There is a locally flat topological embedding $f: S^{3} \rightarrow S^{5}$ which is not equivalent to a smooth embedding; i.e., there exists no homeomorphism $g$ of $S^{5}$ such that $g \circ f$ is smooth.

REMARKs. 1. $S^{5}-f\left(S^{3}\right)$ cannot be the homotopy type of $S^{1}$, by Stallings' unknotting theorem [S].

2. Of course, $f$ is not equivalent to a locally flat piecewise linear embedding either; but since $\pi_{3}$ (Top/PL) $=\pi_{3}$ (Top $\left./ 0\right)$, the obstruction is the same for smoothing [L].

3. The smoothing $\left(S^{3} \times R^{2}\right)_{\alpha}$ induced by $f: S^{3} \times R^{2} \rightarrow S^{5}$ is not diffeomorphic to $S^{3} \times R^{2}$ by results of Kirby and Siebenmann. On the other hand, by taking a smooth embedding of $S^{1}$ in $S^{5}-f\left(S^{3} \times 0\right)$, representing a generator of $H_{1}\left(S^{5}-f\left(S^{3} \times 0\right)\right)$, we have a smooth embedding $f:\left(S^{3} \times R^{2}\right)_{\alpha} \rightarrow S^{5}$-normal tube of $S^{1}=\left(S^{3} \times R^{2}\right)_{0}$; where $\left(S^{3} \times R^{2}\right)_{0}$ is the standard smoothing.

4. Since $\alpha$ is of order 2 , the embedding $f: S^{3} \times R^{2} \rightarrow S^{3} \times R^{2}$ given in Remark 3 induces $\left(S^{3} \times R^{2}\right)_{0}$ from $\left(S^{3} \times R^{2}\right)_{\alpha}$; i.e., there is a smooth embedding of $S^{3}$ in $\left(S^{3} \times R^{2}\right)_{\alpha}$ which is a homotopy equivalence.

It follows that there is also a smooth embedding of $S^{3}$ in $\left(S^{3} \times T^{2}\right)_{\alpha}$, $T^{2}$ the 2-torus, which represents a generator of $\pi_{3}\left(S^{3} \times T^{2}\right)$.

5. There appears to be as many nonsmoothable knots as smoothable ones, since we can take any smooth embedding of $S^{3}$ in $S^{5}$ to define $i$.

\section{BIBLIOGRAPHY}

[CS] S. Cappell and J. Shaneson, On four-dimensional surgery and applications, Princeton University, Princeton, N. J., 1971. (mimeograph).

[KS] R. C. Kirby and L. C. Siebenmann, On the triangulation of manifolds and the Hauptvermutung, Bull. Amer. Math. Soc. 75 (1969), 742-749. MR 39 \#3500.

[K] R. Kirby, "Codimension-two locally flat embeddings," Topology of Manifolds Edited by J. C. Cantrell and C. H. Edwards, Jr., Markham, Chicago, Ill., 1970.

[L] R. Lashof, The immersion approach to triangulation and smoothing, Aarhus Universitet, 1970 (mimeograph). Also: Proc. Sympos. Pure Math., vol. 22, Amer. Math. Soc., Providence, R. I. (to appear).

[S] J. R. Stallings, On topologically unknotted spheres, Ann. of Math. (2) 77 (1963), 490-503. MR $26 \# 6946$.

University of Chicago, Chicago, Illinois 60637 